\title{
ARTICLE An openable artificial intestinal tract system for the in vitro evaluation of medicines
}

\author{
Satoshi Konishi ${ }^{1,3,4}$, Takuya Fujita ${ }^{2,3}$, Koji Hattori ${ }^{3}$, Yusuke Kono ${ }^{2}$ and Yoshifumi Matsushita ${ }^{4}$
}

In vitro drug screening systems for pharmacological targets have been studied as substitutes for whole-animal experiments. Cultured cells or tissues provide promising substitution models when coupled with technological innovations in micro total analysis systems. In this study, we focus on an intestinal drug absorption assay, as the oral route is most frequently used for drug administration. Pharmacological studies have reported the development of artificial vessels that include tubular structures. However, it is difficult to observe the insides of these tubes in situ. To address this problem, we developed a micro-device that uses a pneumatic balloon actuator (PBA) to open and close an artificial intestinal tract. A human colon carcinoma cell line (Caco-2) was cultivated on the flat surface of the micro-device for 7 days to form the inner cellular layer of an artificial intestinal tract with which to evaluate drug transport. The artificial intestinal tract was completely actuated from a flat plate to a circular tube via a PBA with a pressure of $65 \mathrm{kPa}$, and drugs were perfused at a flow rate of $0.05 \mathrm{~mL} \mathrm{~min}{ }^{-1}$ into the tubular artificial intestinal tract for $1 \mathrm{~h}$. Using the openable artificial intestinal tract, the in vitro absorption of calcein and Texas Red were successfully estimated as models of hydrophilic and hydrophobic drugs, respectively. The artificial intestinal tract enables the effective evaluation of the in vitro intestinal absorption of drug candidates and contributes to the reduction of costs incurred during the initial stage of drug development.

Keywords: Caco-2 cells; drug absorption; intestinal tract; microfluidic device; pneumatic balloon actuator

Microsystems \& Nanoengineering (2015) 1, 2015015; doi:10.1038/micronano.2015.15; Published online: 17 August 2015

\section{INTRODUCTION}

In the fields of drug discovery and development, pharmacological studies are conducted to evaluate the performance of new drug candidates. In vitro pharmacological studies, whole-animal studies, and clinical trials are all employed to develop new drugs. Animal studies are performed before clinical trials to guarantee the effectiveness and safety of a drug. Recently, the development of an in vitro drug screening system that can be used instead of whole-animal experiments has been of interest. Cultured cells or tissues are already promising substitution models, and related technologies such as biochips have improved remarkably since they were first introduced. Using micro total analysis system technology, various functional micro-fluidic systems have been developed to evaluate biochemical samples. However, dishes or biochips provide planar cell culture conditions with essential differences from in vivo three-dimensional tissue structures. It is important to establish a model that is closer to actual in vivo conditions for reliable pharmacological tests. Several studies of three-dimensional cell culture, including those based on cellular aggregate technology and cell sheet engineering, have overcome the limitations of these inherent differences ${ }^{1-4}$. Additionally, several studies have reported the development of organs-on-achip $^{5-8}$.

In this paper, we present an artificial intestinal tract as a new in vitro evaluation system for intestinal drug absorption. Intestinal absorption is a representative in vivo drug absorption model used in pharmacokinetic analyses. Figure 1 illustrates an intestine with a hollow tubular structure and its magnified internal structure. The use of a Transwell ${ }^{\varpi}$ cell culture insert (Corning Incorporated Life Sciences, Corning, NY, USA) is common to estimate the intestinal absorption of drug candidates. The Transwell system consists of a transparent framework with multi-well and planar membrane filters as cell growth substrates, but the intestinal tract has a tubular structure. This presents the same problems as those associated with the differences between a two-dimensional in vitro system and three-dimensional tissues. Several technologies have been developed to generate tubular structures, such as artificial blood vessels, for cell-based experiments ${ }^{9,10}$.

It is difficult to observe the inside of a tubular structure, and once cultured cells are sampled or observed by breaking or opening a tubular structure, the cells cannot return to their former states. To address this problem, we propose a novel micro-device that provides an intestine-like hollow tubular structure. Figure 2 represents the proposed micro-device as an artificial intestinal tract that transforms in shape from a flat structure to a tubular structure. This micro-device is the first to enable the in situ observation of cell states within a tube by transforming from a flat structure to a tubular structure. A human colon carcinoma cell line (Caco-2) was used to prepare the artificial intestinal tract in this study. Caco-2 cells were cultured on the flat surface of the micro-device, corresponding to the inner surface of the artificial intestinal tract. We employed the bending motion of a pneumatic balloon actuator (PBA) to transform the artificial intestinal tract.

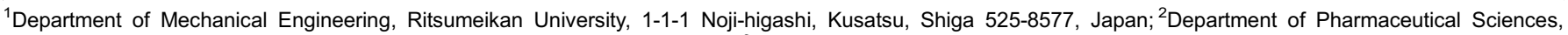

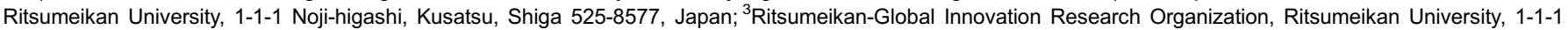

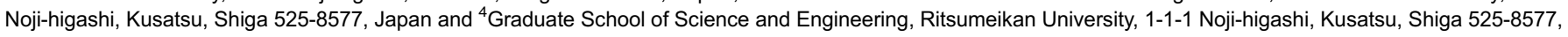
Japan
}

Correspondence: Satoshi Konishi (konishi@se.ritsumei.ac.jp)

Received: 11 May 2015; revised: 17 June 2015; accepted: 25 June 2015 


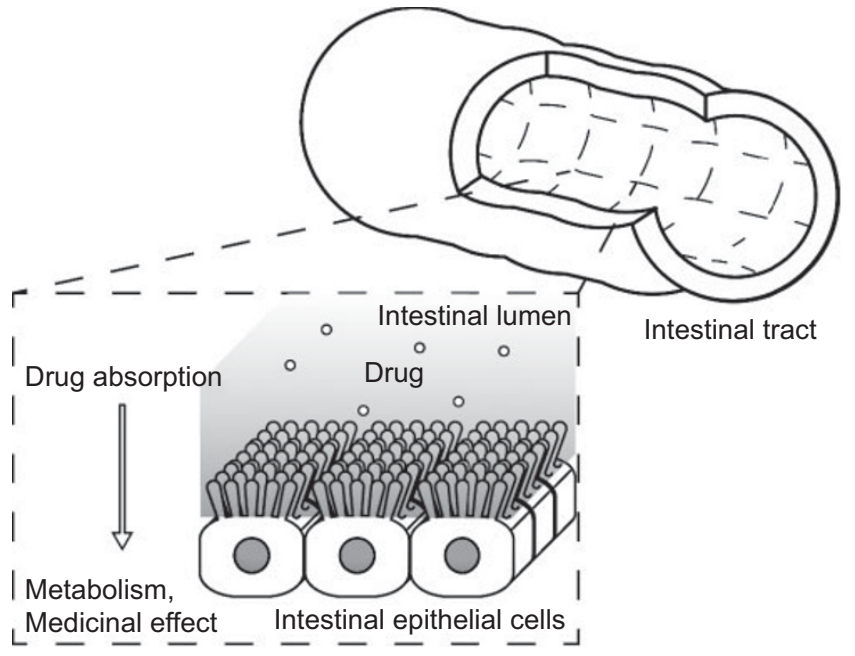

Figure 1 Drug absorption by Caco-2 cells in the intestinal tract.

The PBA has been continuously improved since it was first developed ${ }^{11}$. PBAs used currently, corresponding to the third generation, comprise a balloon formed by two thin polydimethylsiloxane (PDMS) films ${ }^{12}$. Photolithography-based micromachining technology is used to fabricate the fine structures of the balloon. Two PDMS films of PBA are designed to differ in thickness or stiffness to generate unequal stretching during internal pressurization. The unequal stretching of the two films converts the swelling motion to a bending motion, which is based on a bimorph mechanism. The PBA provides a soft and flexible structure and a safe driving principle, properties that are highly compatible with the requirements for devices used in biomedical applications. Various biomedical devices, such as retractors for endoscopic surgery, have been developed using PBAs. A retractor can remove an obstacle (e.g., an organ) to create space in front of an endoscope. A cylindrical micro-structure that was transformed from a planar structure by a PBA array has also been used as a cell sheet transplantation tool for the eye ${ }^{13}$. In this study, we applied PBA technology to develop an openable artificial intestinal tract based on a cylindrical micro-structure.

An artificial intestinal tract can be usefully functionalized by a perfusion system that supplies culture medium and drugs. We connected an external fluidic system and the artificial intestinal tract to construct a perfusion system. The generation of bubbles within the tract causes undesirable medium stagnation and obstructs the observation of cells; we present solutions to these problems related to the implementation of the system.

\section{MATERIALS AND METHODS}

\section{Materials}

Negative photoresists were obtained from MicroChem (Product number: SU-8 3050; Newton, MA, USA). The PDMS prepolymer and its curing agent were obtained from Dow Corning (Product name: Sylgard 184; Midland, MI, USA). The adhesive reagent for the silicone resin was obtained from Cemedine (Product name: SuperX Clear; Tokyo, Japan). Parylene-C was obtained from Specialty Coating Systems (Indianapolis, IN, USA). The polyethylene terephthalate (PET) film was obtained from Toray (Product name: Lumirror; Tokyo, Japan). Polyimide adhesive sealing tape for heat-resistant insulation was obtained from Nitto Denko (Product name: Kapton ', No. 360; Osaka, Japan). Calcein (Mw: 622.54) and Texas Red (Mw: 728.83) as model drugs, calcein-AM, Dulbecco's modified Eagle's medium (DMEM), penicillin G, streptomycin, nonessential amino acids, and trypsin-EDTA were purchased from

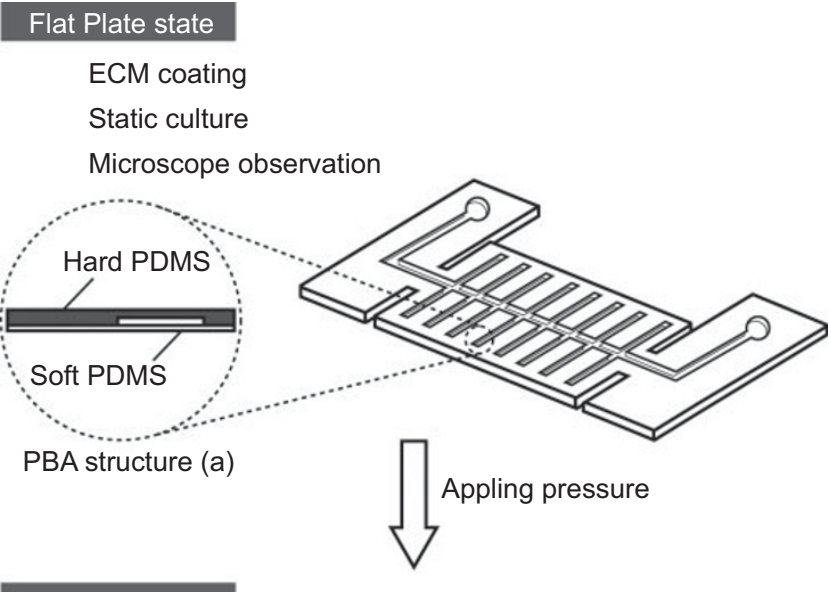

Circular tube state

Medium perfusion

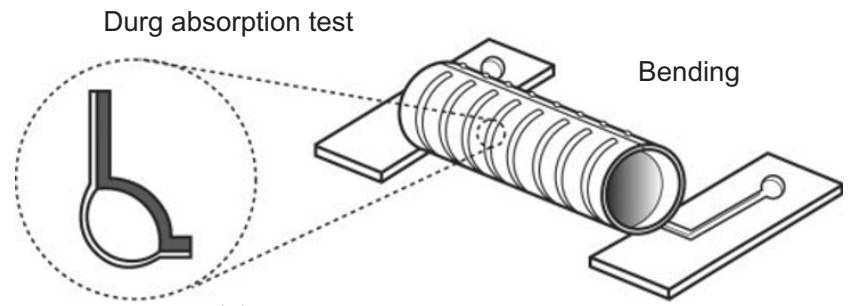

PBA structure (b)

Figure 2 Artificial intestinal tract with a pneumatic balloon actuator. Principle of bending motion: cross-sectional structure of the pneumatic balloon actuator (PBA) without pneumatic pressure (a) and with pneumatic pressure (b).

Nacalai Tesque (Kyoto, Japan). Fetal bovine serum was purchased from Life Technologies Japan (Tokyo, Japan). Methanol was obtained from Wako Pure Chemical Industries Ltd. (Osaka, Japan). Hanks' balanced salt solution (HBSS) and rat tail collagen were obtained from Sigma-Aldrich (St. Louis, MO, USA).

\section{Design}

The artificial intestinal tract consists of PBA, a microchannel for applying air pressure, two seal areas, and two air inlets (Figure 3). The PBA transitions gradually from a flat plane to a circular tube in response to the air pressure delivered through the microchannel. The working principle of PBA has been described in our previous papers ${ }^{11,12}$. After the device is actuated, the circular tube is sealed by fitting seal areas to allow the perfusion of culture media. Table 1 shows the dimensions and calculated hydrodynamic parameters of the artificial intestinal tract. The longer side of the balloon $(d)$ corresponds to the circumference of the actuated artificial intestinal tract. We set a length $d$ of $3.6 \mathrm{~mm}$, and the diameter of the actuated artificial intestinal tract was therefore 1.1 $\mathrm{mm}$. The flow rate of the drug solution was set at $0.05 \mathrm{~mL} \mathrm{~min} \mathrm{~m}^{-1}$ based on these dimensions. Under these flow conditions, the calculated pressure drop in the circular tube is very low, the flow of the drug solution is laminar $(\operatorname{Re}<2100)$, and the shear stress on the inner surface of the circular tube is sufficiently low for cell adhesion $^{14}$.

\section{Microfabrication}

The artificial intestinal tract was fabricated by photolithography and PDMS replica molding ${ }^{15,16}$. The master template for the microfluidic network was fabricated using photolithography. The 


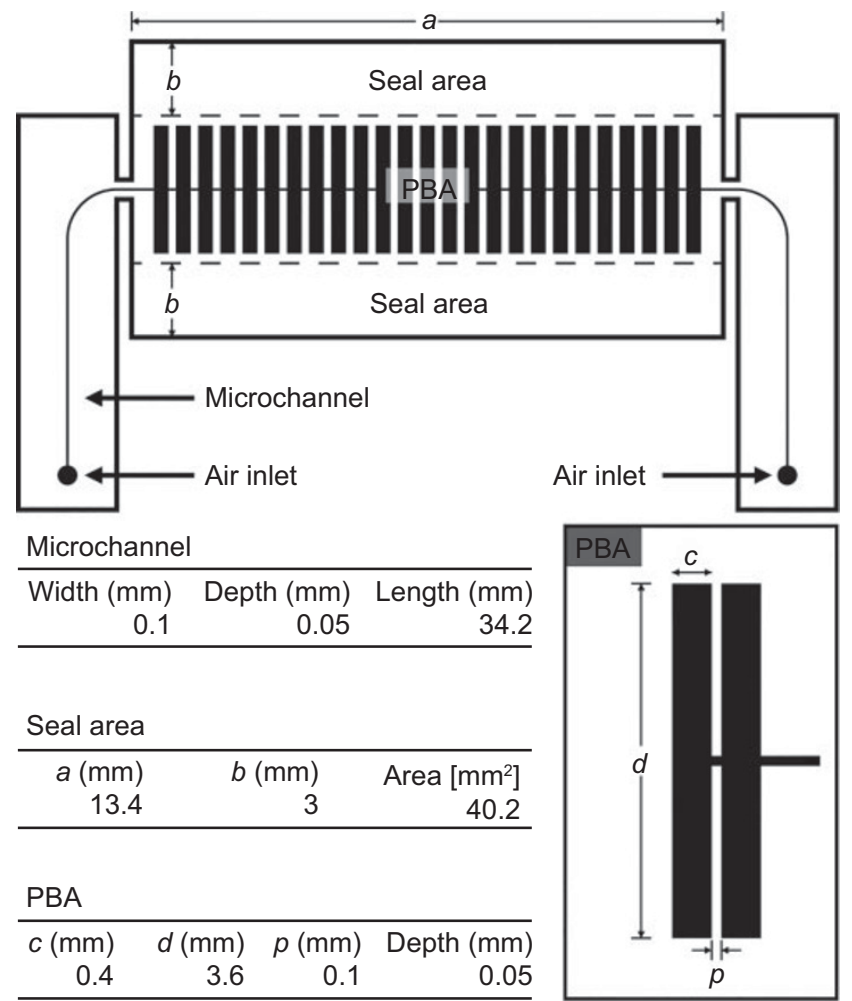

Figure 3 Overhead view, layout, and dimensions of the artificial intestinal tract with a pneumatic balloon actuator.

Table 1 Hydrodynamic parameters of the artificial intestinal tract

\begin{tabular}{lc}
\hline Diameter of circular tube $(\mathrm{mm})$ & 1.1 \\
Length of circular tube $(\mathrm{mm})$ & 13.4 \\
Flow rate $\left(\mathrm{mL} \mathrm{min}^{-1}\right)$ & 0.05 \\
Average flow velocity $\left(\mathrm{mm} \mathrm{s}^{-1}\right)$ & 0.8 \\
Retention time $(\mathrm{s})$ & 13.4 \\
Re $(-)$ & 1.3 \\
Shear stress $(\mathrm{Pa})$ & $10^{-3}$ \\
Pressure drop $(\mathrm{Pa})$ & 0.2 \\
\hline
\end{tabular}

photolithography process consists of spin-coating, soft-baking, exposure, and post-exposure baking. SU-8 3050 was spin-coated onto a silicon wafer at a thickness of approximately $50 \mu \mathrm{m}$ and then baked (soft-baking: $65{ }^{\circ} \mathrm{C}$ for $3 \mathrm{~min}$ and $95^{\circ} \mathrm{C}$ for $6 \mathrm{~min}$ ). To create a microchannel pattern for the balloon actuator, the photoresist layer was exposed to UV light $(365 \mathrm{~nm}, 9.0 \mathrm{~mW}$ $\mathrm{cm}^{-2}$ ) for $9 \mathrm{~s}$ through the photomask and then baked (postbaking: $95{ }^{\circ} \mathrm{C}$ for $7 \mathrm{~min}$ ). The microchannel pattern for PBA was developed using SU-8 developer for 5 min. Parylene-C was coated on the master template by vapor-deposition for mold release in the subsequent PDMS replica molding process (thickness: $<0.1$ $\mu \mathrm{m})$. The PDMS prepolymer and curing agent were mixed at an $8: 1$ ratio and spin-coated on the master template at a thickness of approximately $90 \mu \mathrm{m}$. After curing in an oven at $85^{\circ} \mathrm{C}$ for $20 \mathrm{~min}$, the micropatterned PDMS layer was carefully peeled away from the master template. The PDMS prepolymer and curing agent were also mixed at a 12:1 ratio and spin-coated on the silicon wafer without photoresist patterns at a thickness of approximately $20 \mu \mathrm{m}$. After curing at $65^{\circ} \mathrm{C}$ for $5 \mathrm{~min}$, the PDMS flat layer was fabricated. The PDMS flat layer was imperfectly cured and placed in contact with the micropatterned PDMS layer. The

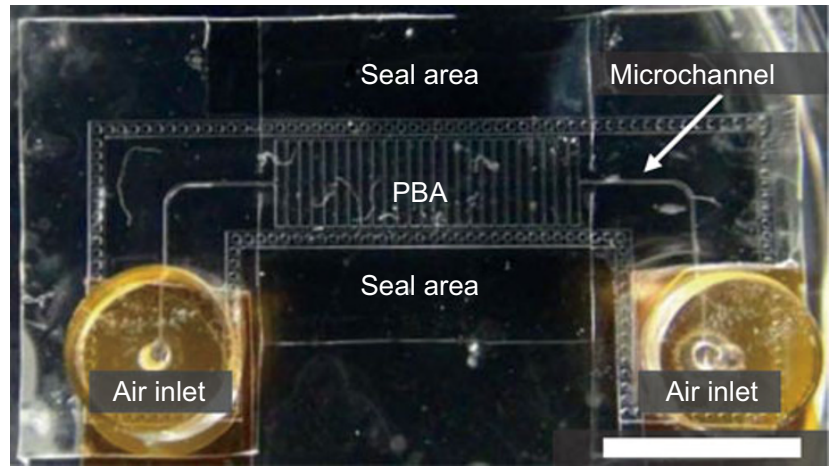

Figure 4 Photograph of the fabricated artificial intestinal tract with pneumatic balloon actuator. Scale bar: $1 \mathrm{~cm}$.

assembled PDMS layer was perfectly cured at $85^{\circ} \mathrm{C}$ for $10 \mathrm{~min}$. After curing, the adhered PDMS layers in contact with the silicon wafer were washed by ultrasonication in ethanol for $1 \mathrm{~min}$ and dried for $12 \mathrm{~h}$.

\section{Surface modification for device actuation}

PDMS is highly permeable to air, but PBA requires low air permeability to maintain internal pressure. In this study, we modified the surface of the balloon area on the PDMS device with parylene to decrease air permeability. Before applying this surface modification, the seal area on the PDMS device was physically masked with PET film. The balloon area on the masked device was coated with parylene- $C$ via vapour deposition (thickness: 0.1 $\mu \mathrm{m})$. After the parylene coating was applied, the physical mask was peeled away from the device.

\section{Assembly}

The cylindrical connector fabricated from PDMS was bonded to each air inlet using an adhesive reagent. Through-holes $(\phi 1 \mathrm{~mm})$ that enabled the application of air pressure to the microchannel were opened at the connectors using a hollow punch, and through-holes on the opposite sides of the connectors were sealed using sealing tape (Figure 4).

\section{Surface modification for cell culture}

Collagen is often used as an extracellular matrix for Caco-2 monolayer cultures and was physically adsorbed on the surface of the parylene-coated device before cell culture began. The parylene-coated device was sterilized by UV irradiation for $1 \mathrm{~h}$ using a germicidal lamp. After UV sterilization, a spacer (opening dimensions: $0.4 \mathrm{~cm} \times 1.0 \mathrm{~cm}, 3 \mathrm{~mm}$ thickness) fabricated from PDMS was placed on the balloon area (i.e., the culture area). The balloon area within the spacer was treated with methanol for 12 $\mathrm{h}$ to make its surface hydrophilic. Then, $0.1 \mathrm{mg} \mathrm{mL}^{-1}$ collagen solution $(100 \mu \mathrm{L})$ was poured into the spacer and collagen was physically adsorbed onto the hydrophilic surface for $1 \mathrm{~h}$. The collagen layer, which acts as a scaffold for cell attachment, formed on the device without gelation. The fully formed collagen layer was several nanometres thick.

\section{Cell culture}

Caco-2 cells are the most commonly used cells for in vitro intestinal absorption studies ${ }^{17}$. The human intestinal cell line Caco-2 was obtained from DS Pharma Biomedical Co, Ltd. (Osaka, Japan). Caco-2 cells were cultured in DMEM supplemented with $10 \%$ heat-inactivated fetal bovine serum, penicillin G (100 U $\left.\mathrm{mL}^{-1}\right)$, streptomycin $\left(100 \mu \mathrm{g} \mathrm{mL}^{-1}\right)$, and $1 \%$ non-essential amino 
acids at $37{ }^{\circ} \mathrm{C}$ in $5 \% \mathrm{CO}_{2}-95 \%$ air. Caco-2 cells were passaged by dissociation in $0.05 \%$ trypsin and EDTA, and the cells $\left(1 \times 10^{4}\right)$ were seeded on the collagen-coated inner surface of the artificial intestinal tract. The Caco- 2 cells reached confluence 7 days later. The culture medium was replaced every $24 \mathrm{~h}$. The formation of the Caco-2 monolayer was observed using an IX81 motorized inverted system microscope (Olympus, Tokyo, Japan).

\section{Influence of device actuation on cellular adhesion}

The influence of device actuation on cellular adhesion and viability was evaluated by dyeing the Caco- 2 monolayer with calcein-AM. Calcein-AM is a membrane-permeable nonfluorescent dye that is converted to green fluorescent calcein by intracellular esterase hydrolysis in live cells. After the formation of the Caco-2 monolayer on the inner surface of the artificial intestinal tract, the monolayer was exposed to calcein-AM $\left(1 \mu \mathrm{g} \mathrm{mL}^{-1}\right)$ for $1 \mathrm{~h}$. Then, the device was actuated from a flat plate to a circular tube for 10 s. The Caco-2 monolayer was observed using an IX81 microscope with bright field illumination and a fluorescent imaging unit (U-MNIBA2) before and after actuation of the device, and this procedure was repeated 10 times.

\section{Absorption study of fluorescent dyes in the artificial intestinal tract}

After the formation of a monolayer of Caco- 2 cells, the artificial intestinal tract was actuated from a flat plane to a circular tube. HBSS containing two fluorescent dyes, calcein and Texas Red, were perfused in the artificial intestinal tract for $1 \mathrm{~h}$. Calcein and Texas Red were used as models of hydrophilic and hydrophobic drugs, and their concentrations were $100 \mu \mathrm{mol} \mathrm{L}^{-1}$ and $10 \mu \mathrm{mol} \mathrm{L} \mathrm{L}^{-1}$,

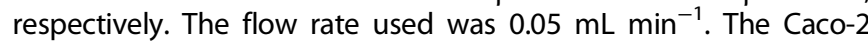
monolayer was washed via HBSS perfusion of the artificial intestinal tract for $5 \mathrm{~min}$ (flow rate: $0.05 \mathrm{~mL} \mathrm{~min}^{-1}$ ). The adsorption of fluorescent dyes in cells was observed using an IX81 microscope with a fluorescent imaging unit (U-MNIBA2 and U-MWIG2).

\section{RESULTS AND DISCUSSION}

\section{Evaluation of the bending motion of the artificial intestinal tract}

We observed the actuation of the artificial intestinal tract and found that PBA bending was successfully generated through the application of pressure. At $65 \mathrm{kPa}$ of air pressure, the artificial intestinal tract was completely actuated from a flat plane to a circular tube without air leakage (Figure 5).

\section{Constitution of the artificial intestinal tract}

Figure 6a shows the surface of the artificial intestinal tract without Caco- 2 cells. Caco- 2 cells attached to the surface of the culture area (Figure 6b), and a Caco-2 monolayer formed successfully (upper layer of PBA). In addition, no detachment of Caco-2 cells was observed after 10 repetitions of device actuation (Figure $6 \mathrm{c}$ and e). Cell adhesion and viability were also evaluated using calcein-AM. The Caco-2 monolayer on the artificial intestinal tract was homogeneously dyed by calcein-AM, independent of device actuation (Figure $6 \mathrm{~d}$ and $\mathrm{f}$ ). These results demonstrate that cell viability was maintained and the Caco- 2 monolayer was tightly attached during repeated actuations of the device. Homogeneous dyeing with calcein-AM was observed as shown in Figure $6 \mathrm{~d}$ and e. These results indicate that the Caco- 2 monolayer is tightly formed by the intercellular junctions between cells. The cellular adhesion of Caco-2 cells on the culture area was sufficiently strong to maintain the cell monolayer during PBA expansion. a

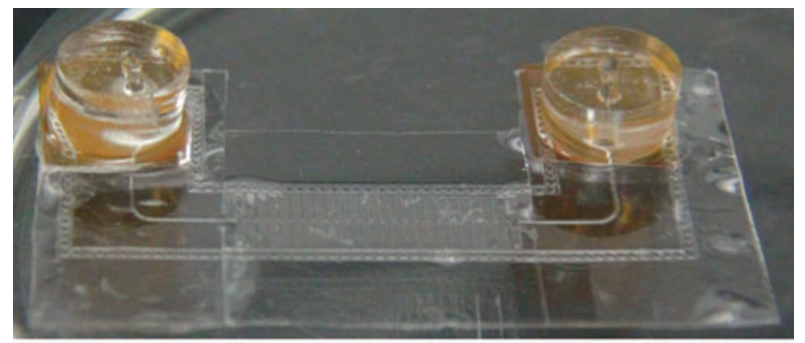

b

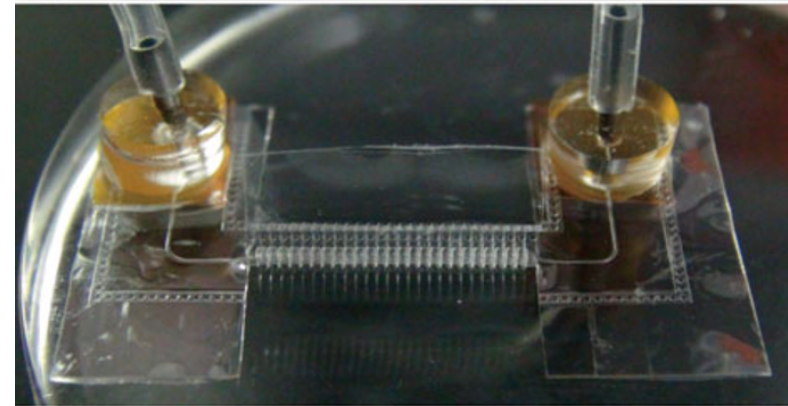

c

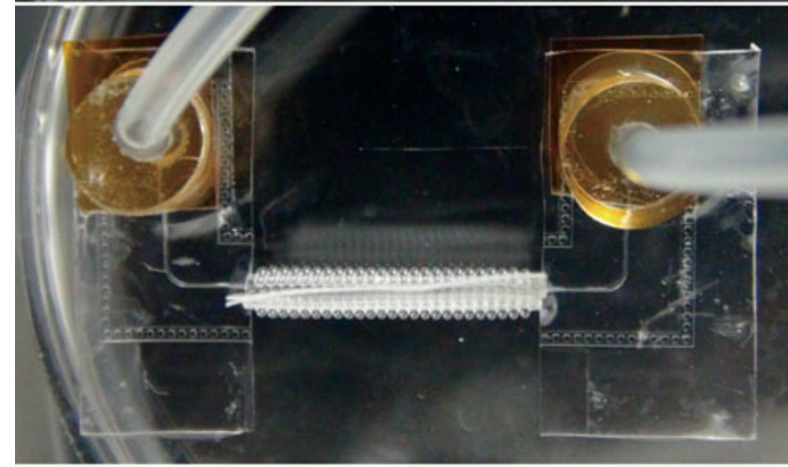

d

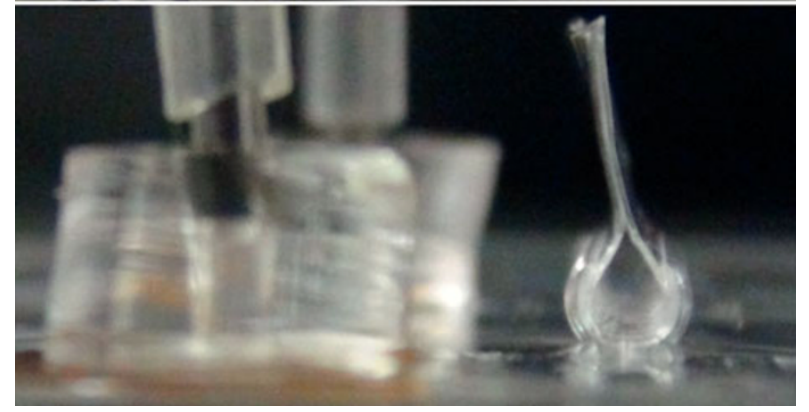

Figure 5 Bending motion of the pneumatic balloon actuator. (a) Front-view before bending, (b) front-view, (c) top-view, and (d) sideview after bending.

\section{Evaluation of drug absorption in Caco-2 cells using the artificial intestinal tract}

Figure 7 shows the flow system used for drug absorption tests in the artificial intestinal tract. To investigate the absorptive characteristics of Caco- 2 cells cultured on the inner surface of the artificial intestinal tract, a drug solution with calcein and Texas Red was flowed into the artificial intestinal tract for $1 \mathrm{~h}$. During the drug adsorption test, no leakage from any of the connections in the flow system was observed under the flow conditions used (Table 1).

Figure 8 shows microscopy images of the Caco-2 monolayer on the inner surface of the artificial intestinal tract after perfusion of the drug solution. Based on the bright-field images, no detachment of Caco-2 cells was observed as a result of shear stress 
a

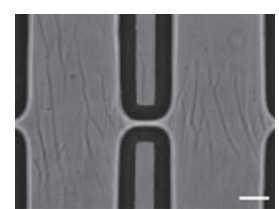

b

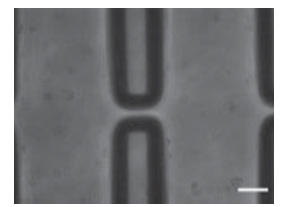

c

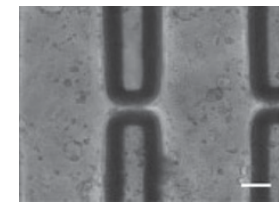

d

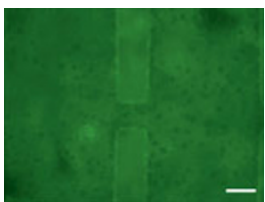

e

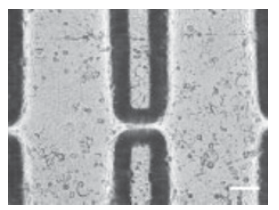

f

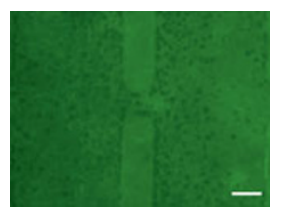

Figure 6 Constitution of an artificial intestinal tract by static cell culture on the device surface, (a) after collagen coating, (b) after cell seeding, (c) after static culture, and (d) after bending of the pneumatic balloon actuator (repeated 10 times). Scale bars: $100 \mu \mathrm{m}$.

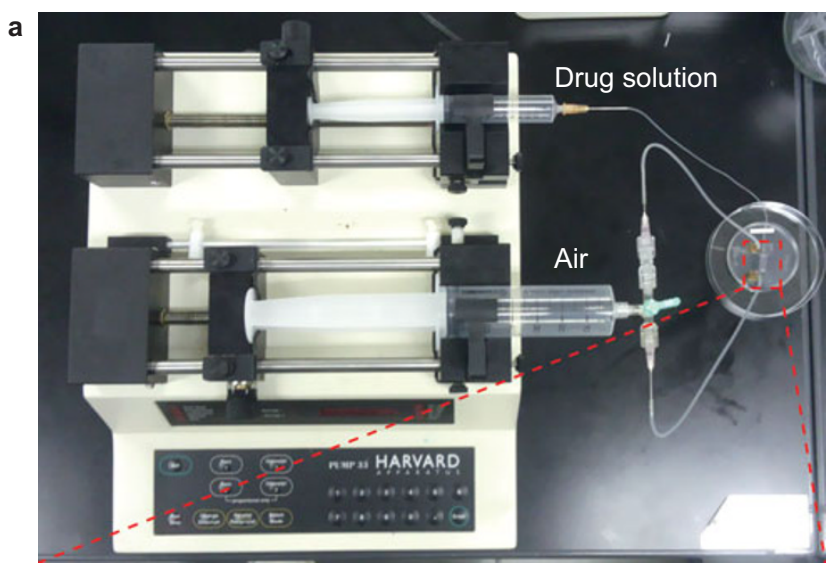

b

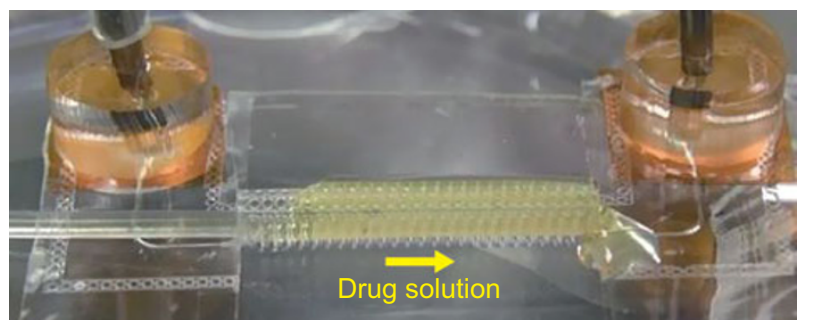

Figure 7 Drug absorption test using the artificial intestinal tract, (a) device configuration, (b) perfusion of drug solution into the artificial intestinal tract.

during perfusion of the drug solution. However, fluorescent signals indicating both calcein and Texas Red were clearly observed, independent of cell position, at the bottom and the upper sides of the artificial intestinal tract. These results suggest that the dye solution homogeneously flowed within the artificial intestinal tract under the flow conditions used (Table 1). Because the Caco-2 monolayer is tightly formed by the intercellular junctions between cells, minimal permeation of dye molecules between the cells was expected. Therefore, the two fluorescent dyes were assumed to be adsorbed homogeneously in the Caco2 monolayer. These dyes were similarly localized in the Caco- 2 monolayer despite their different hydrophilicities. These results suggest that both dyes were transferred through the cellular membrane by passive diffusion, as they were sufficiently small and of comparable sizes. In this case, drug transport was affected

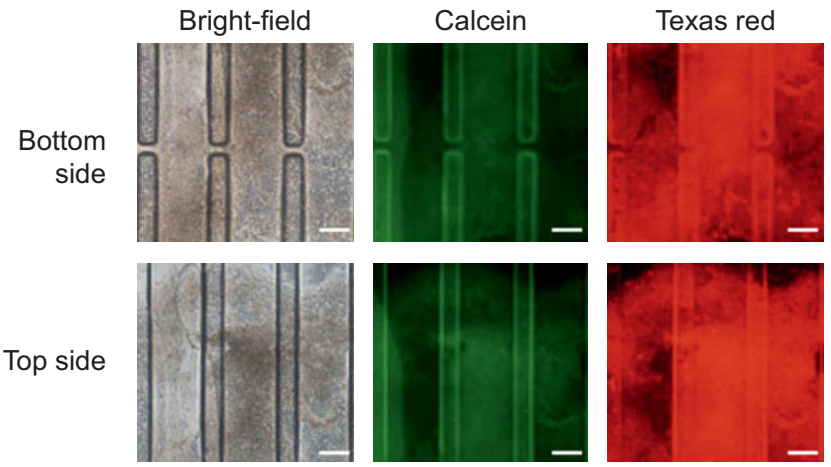

Figure 8 Absorption of fluorescent dyes by Caco-2 cells cultured on the inner surface of the artificial intestinal tract. Scale bars: $200 \mu \mathrm{m}$.

by differences in hydrophilicity, rather than by molecular size. The openable artificial intestinal tract enables the formation of a Caco2 monolayer cultured in a three-dimensional architecture while flat, and the evaluation of cellular morphology and the in vitro absorption of both hydrophilic and hydrophobic drugs in a tubular conformation.

We developed an artificial intestinal tract for use in pharmacological tests that can be actuated from a flat plane to a circular tube by PBA. We evaluated the adsorption of drugs with different hydrophilicities by a Caco-2 monolayer that formed homogeneously on the inner surface of this artificial intestinal tract. The results regarding intestinal absorption tend to differ between in vivo (clinical) and in vitro (cell culture) tests ${ }^{18,19}$. This is due to the presence of a mucosal layer secreted by epithelial cells on the surface of the Caco-2 monolayer. Mucous is secreted by intestinal epithelial cells and maintained on the surface of the Caco-2 monolayer during drug adsorption ${ }^{20}$. In pharmacokinetic studies, the secreted mucous layer is often referred to as an unstirred water layer. Because water-soluble drugs freely diffuse in the secreted mucous layer, water-soluble calcein can easily contact the surface of the Caco-2 monolayer and is efficiently absorbed by the inner surface of the artificial intestinal tract. However the secreted mucous layer behaves as a permeability barrier against lipophilic drugs, and the secreted mucous layer on the surface of a Caco-2 monolayer is substantially thicker than that of the in vivo intestinal tract ${ }^{21}$. Therefore, the absorption of lipophilic drugs is restricted in Caco-2 monolayers under static conditions. Mass transfer occurs mainly by passive diffusion in the secreted mucous layer, and mass transfer resistance depends on the flow rate of the drug solution ${ }^{22,23}$. In this study, we observed that the absorption of lipophilic Texas Red into the Caco-2 monolayer was higher than that of calcein. These results suggest that the resistance of the secreted mucous layer is decreased by perfusion of the drug solution within the artificial intestinal tract. The artificial intestinal tract enables the effective evaluation of the in vitro intestinal absorption of drug candidates and contributes to a reduction in the costs incurred during the initial stage of drug development.

\section{ACKNOWLEDGEMENTS}

This work was conducted as part of the Ritsumeikan Global Innovation Research Organization (R-GIRO) project at Ritsumeikan University and was supported by JSPS KAKENHI (Grant-in-Aid for Challenging Exploratory Research, Grant No. 15K12526).

\section{COMPETING INTERESTS}

The authors declare no financial or commercial conflicts of interest. 


\section{REFERENCES}

1 Spence JR, Mayhew CN, Rankin SA et al. Directed differentiation of human pluripotent stem cells into intestinal tissue in vitro. Nature 2011; 470: 105-U120.

2 Lancaster MA, Knoblich JA. Organogenesis in a dish: Modeling development and disease using organoid technologies. Science 2014; 345: 10.

3 Haraguchi Y, Shimizu T, Sasagawa $T$ et al. Fabrication of functional threedimensional tissues by stacking cell sheets in vitro. Nature Protocols 2012; 7: 850-858.

4 Takahashi H, Shimizu T, Nakayama M et al. The use of anisotropic cell sheets to control orientation during the self-organization of 3D muscle tissue. Biomaterials 2013; 34: 7372-7380.

5 Shintu L, Baudoin R, Navratil V et al. Metabolomics-on-a-chip and predictive systems toxicology in microfluidic bioartificial organs. Analytical Chemistry 2012; 84: $1840-1848$.

6 Huh D, Matthews BD, Mammoto A et al. Reconstituting organ-level lung functions on a chip. Science 2010; 328: 1662-1668.

7 Moraes C, Mehta G, Lesher-Perez SC et al. Organs-on-a-chip: A focus on compartmentalized microdevices. Annals of Biomedical Engineering 2012; 40: 1211-1227.

8 Wikswo JP, Curtis EL, Eagleton ZE et al. Scaling and systems biology for integrating multiple organs-on-a-chip. Lab on a Chip 2013; 13: 3496-3511.

9 Yalcin O, Ulker P, Yavuzer $U$ et al. Nitric oxide generation by endothelial cells exposed to shear stress in glass tubes perfused with red blood cell suspensions: Role of aggregation. American Journal of Physiology-Heart and Circulatory Physiology 2008; 294: H2098-H2105.

10 Bergh N, Ekman M, Ulfhammer E et al. A new biomechanical perfusion system for ex vivo study of small biological intact vessels. Annals of Biomedical Engineering 2005; 33: 1808-1818.

11 Konishi S, Kawai F, Cusin P. Thin flexible end-effector using pneumatic balloon actuator. Sensors and Actuators A-Physical 2001; 89: 28-35.

12 Jeong OC, Konishi S. All PDMS pneumatic microfinger with bidirectional motion and its application. Journal of Microelectromechanical Systems 2006; 15: 896-903.

13 Tokida M, Obara T, Takahashi $\mathrm{M}$ et al. Integration of cell sheet sucking and tactile sensing functions retinal pigment epithelium transplantation tool. IEEE 23rd International Conference on Micro Electro Mechanical Systems (IEEE MEMS 2010); Hong Kong, China; 24-28 Jan 2010; 2010: 316-319.
14 Kim HJ, Huh D, Hamilton $\mathrm{G}$ et al. Human gut-on-a-chip inhabited by microbial flora that experiences intestinal peristalsis-like motions and flow. Lab on a Chip 2012; 12: 2165-2174.

15 Duffy DC, McDonald JC, Schueller OJA et al. Rapid prototyping of microfluidic systems in poly(dimethylsiloxane). Analytical Chemistry 1998; 70: 4974-4984.

16 Deng T, Wu HK, Brittain ST et al. Prototyping of masks, masters, and stamps/ molds for soft lithography using an office printer and photographic reduction. Analytical Chemistry 2000; 72: 3176-3180.

17 Shah P, Jogani V, Bagchi T et al. Role of Caco-2 cell monolayers in prediction of intestinal drug absorption. Biotechnology Progress 2006; 22: 186-198.

18 Yang YS, Faustino PJ, Volpe DA et al. Biopharmaceutics classification of selected beta-blockers: solubility and permeability class membership. Molecular Pharmacology 2007; 4: 608-614.

19 Irvine JD, Takahashi L, Lockhart K et al. MDCK (Madin-Darby canine kidney) cells: A tool for membrane permeability screening. Journal of Pharmaceutical Sciences 1999; 88: 28-33.

20 Cone RA. Barrier properties of mucus. Advanced Drug Delivery Reviews 2009; 61: 75-85.

21 Korjamo T, Heikkinen AT, Monkkonen J. Analysis of unstirred water layer in in vitro permeability experiments. Journal of Pharmaceutical Science 2009; 98: 4469-4479.

22 Karlsson J, Artursson P. A method for the determination of cellular permeability coefficients and aqueous boundary-layer thickness in monolayers of intestinal epithelial (Caco-2) cells grown in permeable filter chambers. International Journal of Pharmaceutics 1991; 71: 55-64.

23 Hillgren KM, Kato A, Borchardt RT. In-vitro systems for studying intestinal drug absorption. Medicinal Research Reviews 1995; 15: 83-109.

This work is licensed under a Creative Commons Attribution 4.0 Unported License. The images or other third party material in this article are included in the article's Creative Commons license, unless indicated otherwise in the credit line; if the material is not included under the Creative Commons license, users will need to obtain permission from the license holder to reproduce the material. To view a copy of this license, visit http://creativecommons. org/licenses/by/4.0 